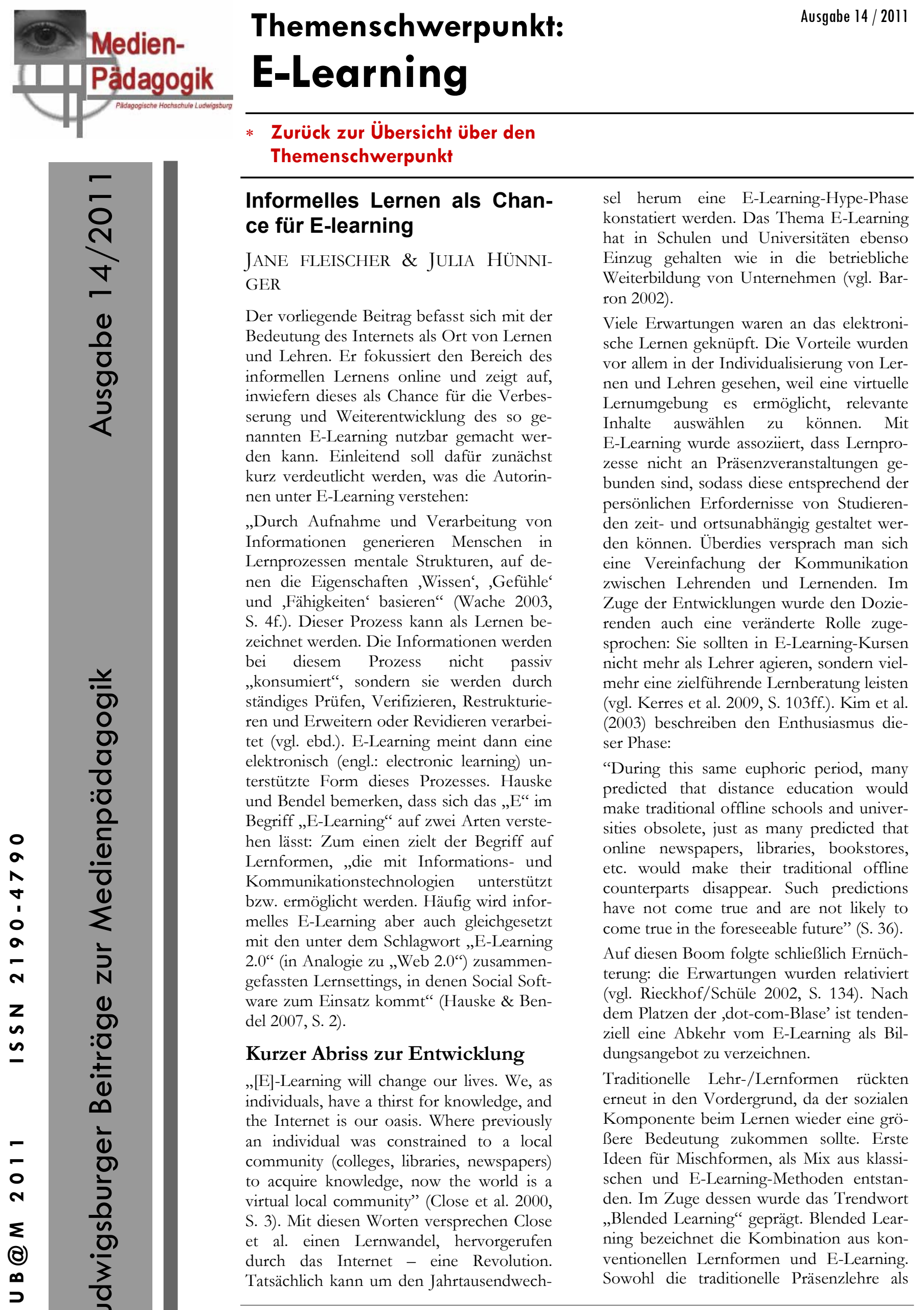


auch E-Learning bieten verschiedene Gestaltungsmöglichkeiten. Durch eine sinnvolle Verknüpfung dieser Lernformen kann die Gestaltungsvielfalt noch gesteigert werden (Myrach et al. 2007, S. 197).

\section{Status quo}

Während sich erste E-Learning-Vorhaben an Hochschulen vor allem projektartig und in erster Linie technikorientiert entwickelten, wuchs in den letzten Jahren auch das Bewusstsein dafür, dass nicht nur die technische E-Learning-Infrastruktur zur erfolgreichen Etablierung von E-Learning beiträgt. Mittlerweile ist klar, dass E-Learning-Angebote einem integrierten Ansatz folgen müssen:

„Technik, Organisation und Didaktik sind in diesem Zusammenhang nicht trennbar. Vorhandene Dienste der Hochschulen wie didaktische Zentren, Rechenzentren und Bibliotheken können erfolgreich im Rahmen eines integrierten Serviceangebots gebündelt werden. Das führt dazu, dass die Einrichtungen E-Learning mit einem ganzheitlichen Blick betrachten und ihre Dienste in diesem Gefüge neu abstimmen“" (Albrecht et al. 2007, S. 77).

An vielen Hochschulen entschied man sich zur Implementierung von E-Learning für eine Ausgliederung aus der Universität hin zu externen hochschulübergreifenden Kompetenzzentren (z.B. DIZ Bayern). Diese Auslagerung ermöglichte das Zurückgreifen auf gebündelte Kompetenzen, hatte und hat aber Auswirkungen auf die Nachhaltigkeit der E-LearningMaßnahmen (vgl. Kubicek et al. 2004, S. 16). Die Gründe dafür liegen vorrangig in der mangelnden Akzeptanz von Fremdentwicklungen und technischen Inkompatibilitäten von Plattformen und Dateiformaten.

Nachhaltigkeit ist in der Fachdiskussion zum Thema E-Learning der zentrale Gegenstand. Eine erfolgreiche dauerhafte Implementierung von E-Learning an Hochschulen hängt von vielen Faktoren ab. In der Vergangenheit sind viele E-Learning-Projekte in erster Linie an der Fehlkonzeption von Lernplattformen gescheitert. In der Diskussion um den Zusammenhang von E-Learning, Web 2.0 und Social Media rückt vermehrt die Frage in den Vordergrund, ob der Lerner zur E-LearningPlattform kommen sollte oder das E-Learning zum Lerner (z.B. in sein soziales Netzwerk)? Zauchner et al. (2010, S. 2) bieten darauf eine schlüssige Antwort:

„Technologische Systeme von heute sollten $[\ldots]$ angehalten sein, sich zu öffnen und $\mathrm{Zu}-$ gänge zu neuem Wissen in Form von Wegweisern nach außen zu schaffen. [...] Lernarchitekturen von heute sind daher gefordert, Netzwerke von Lernenden, Lehrenden [und] Inhalten $[. ..] \mathrm{zu}$ etablieren“. Im folgenden
Abschnitt soll nun aus der Perspektive des Internets ein zweiter Blick auf das Lernen junger Menschen geworfen werden.

\section{Das Internet als Bildungs- und Lern- ort}

„Soll das Internet als Raum analysiert werden, der die Möglichkeiten und Fähigkeiten der NutzerInnen erweitern und Bildungszugänge eröffnen kann, so sind zunächst die Bedingungen, unter denen Internetnutzung in der Regel stattfindet, näher zu betrachten“ (Iske et al. 2007, S. 69).

Angesichts eines Überflusses an Informationen fühlen sich viele Menschen heute mit einem Gefühl der Orientierungslosigkeit und der Gefahr der Fehlinformation konfrontiert. Die Herausforderung liegt in der Erschließung der Wissensfülle. Die Lernenden müssen dabei „neue Anforderungen und Aufgaben bewältigen können $[\ldots]$ aber sie sind zunehmend auch selbst zuständig und verantwortlich für die Entwicklung und Weiterentwicklung ihrer Fähigkeiten" (Brinkmann 2000, S. 22).

Hasebrink und Domeyer, die sich mit dem Informationsverhalten von Menschen befassen, unterscheiden in diesem Zusammenhang vier Arten von Informierungsanlässen: Ungerichtete Informationsbedürfnisse, Thematische Interessen, Gruppenbezogene Bedürfnisse und konkrete Problemlösebedürfnisse. Dabei verstehen sie unter einem ungerichteten Informationsbedürfnis die Notwendigkeit den eigenen Lebenskontext permanent zu beobachten, zu bewerten und auf Veränderungen zu überprüfen um neue Chancen und Risiken rechtzeitig wahrzunehmen und so adäquat auf diese reagieren zu können. Eine Informationssuche bezieht sich dann auf ein thematisches Interesse, wenn ihr Ziel eine Vertiefung oder Spezialisierung der sich informierenden Person in Bezug auf ein eingegrenztes inhaltliches Feld ist. Gruppenbezogene Bedürfnisse dienen dem Erleben und der Reflexion desselben innerhalb der verschiedenen Gruppen, in die eine Person eingebunden ist. So entsteht nicht nur eine feste Bindung zu den anderen Gruppenmitgliedern, sondern das Individuum kann sich auch seiner selbst vergewissern. Schließlich sind mit konkreten Problemlösebedürfnissen diejenigen Informationsbedarfe gemeint, die den/die Einzelne/n dazu befähigen auf eine aktuelle Fragestellung oder einen Konflikt angemessen zu reagieren und diese/n zu lösen (vgl. 2010, S. 54f.).

Ausgehend von ihrer Systematik der Informationsbedürfnisse konstatieren die Autoren zwei Wandlungsprozesse bezüglich des Informationsverhaltens von Menschen.

So haben sich diese einerseits im Zeitverlauf gewandelt. Während ab den 70er Jahren die klassischen Massenmedien die primäre Nachrichten- 
quelle für die meisten darstellte und eine Informationsbereitstellung durch den Durchschnittsbürger lediglich in Ausnahmefällen geschah, standen von den Medienmachern gesetzte themenspezifische Informationen im Mittelpunkt. Mit der Differenzierung der Zielgruppen und dem Entstehen immer neuer Sparten wurden themenbezogene Informationsbedürfnisse ab Mitte der 80er Jahre zunehmend besser bedient. Doch erst die fortschreitende Digitalisierung ermöglichte auch die Bedienung gruppenbezogener und problemlösungsorientierter Informationsbedürfnisse (vgl. ebd., S. 59f.). So ermöglichen neue und vielfältigere Kommunikationsmöglichkeiten im Internet (auch im mobilen Netz) einen stetigen Austausch mit immer mehr Mitgliedern der eigenen Bezugsgruppen. Die immer stärkere Verbreitung von Foren ermöglicht heute die Lösung der vielfältigsten Probleme ohne Hilfe so genannter Experten. Mit dem Internet fand aber auch die als ungerichtet bezeichnete Informationssuche stärkeren Zuspruch.

Neben dieser historischen Verschiebung der Informationsbedürfnisse unterstellen Hasebrink und Domeyer auch im Verlauf individueller Biografien einen Wandel im Informationsverhalten. Die Lebensabschnitte Jugend, junges und mittleres Erwachsenenalter sind dementsprechend durch unterschiedliche Foki bei den individuellen Informationsbedarfen gekennzeichnet. Die Autoren beziehen ihre Annahmen auf die Entwicklungsaufgaben, welche jeder Mensch im Laufe seines Lebens bewältigen muss. Daraus schließen sie, dass in der Jugend - der Phase der Identitätsbildung - vor allem die gruppenspezifischen Informationsbedürfnisse im Vordergrund stehen. Im jungen Erwachsenenalter spielen diese zwar weiterhin eine Rolle, es nehmen aber auch die themenspezifischen Informationsbedarfe zu, was aus dem engem Zusammenhang der Wahl eines Berufs mit dieser Lebensphase geschlossen werden kann. Im mittleren Erwachsenenalter, in dem sich Individuen in ihrem Umfeld etablieren, rücken diese Bedürfnisse dann zugunsten der ungerichteten in den Hintergrund (vgl. ebd., S. 59ff.).

Neben den individuellen Änderungen im Informationsverhalten, welches Voraussetzung jeglichen Lernens ist, sind die Zugänge zu Bildung - insbesondere im Bereich des Internets - auch soziokulturell geprägt: „Bei der Nutzung des Internet stehen [...] Nutzungsmotive im Vordergrund, für die, je nach Kontext, die jeweils individuellen Interessen, Präferenzen aber auch Fähigkeiten den Rahmen und Ausgangspunkt darstellen. Diese erscheinen zwar als subjektiv, sind jedoch durch die den NutzerInnen verfügbaren sozialen (Peergroup, Beziehungen, soziale Unterstützung) und kulturellen (Bildungshintergrund,
Wissen, Nutzungskompetenzen) Ressourcen geprägt“ (Iske et al. 2007, S. 85). Entsprechend werden durch verschiedene Personen auch jeweils unterschiedliche Angebote als attraktiv wahrgenommen und genutzt. Iske et al. verweisen in diesem Zusammenhang auf die Problematik der Bestrebung allen Menschen einen Bildungszugang ermöglichen zu wollen, während es durchaus Personengruppen gibt, „die nicht den Anforderungen selbstgesteuerter, themenbezogener und auf eigenverantwortliche Bildungsaneignung setzender Angebote ohne weiteres entsprechen können oder wollen“ (ebd.). Zur Lösung dieser Schwierigkeit merken die Autoren an, dass die Mehrzahl der Nutzungsgelegenheiten, welche das Internet bietet und welche gleichsam Anwendung finden, im informellen Kontext angesiedelt sind. Sie schlagen ein Konzept non-formeller Interventionen in diesen Kontexten vor, welche neben einem dezidierten Qualifikationsziel in Bezug auf die Lerner auch deren Nutzungsgewohnheiten in den Blick nehmen (vgl. ebd.). Bevor die für diesen Zweck vorgestellten Qualitätsanforderungen für entsprechende Interventionen vorgestellt werden, soll überblicksartig auf die Bedeutung der Bildung in informellen Kontexten und auf das informelle Lernen selbst eingegangen werden.

\section{Informelles Lernen und Lernen in informellen Kontexten}

Eines der brisantesten Themen der modernen Wissensgesellschaft ist das lebenslange Lernen. Zur stetigen Realisierung des lebenslangen Lernens erhält die Einbeziehung jeglicher Formen des Lernens besondere Relevanz, denn Lernen ist durchaus eine Überlebensfunktion: „Ohne lebenslange lernende Auseinandersetzung mit dem, was aus der Umwelt, der Lebens-, Arbeits- und Medienwelt, immer wieder an Eindrücken und Anforderungen auf sie zukommt, können Menschen nicht als Person mit eigenem Denken und persönlicher Verantwortung überleben“ (Dohmen 2001, S. 7).

Aus dieser Perspektive erscheint also ein erweitertes Verständnis von Lernen sinnvoll, denn Lernen ereignet sich nicht nur bewusst in dafür vorgesehenen Bildungseinrichtungen. Dohmen schlägt einen weiten holistischen Lernbegriff vor: „Lernen wird danach nicht nur als bewusste kognitive, sondern auch als mehr unbewusste psychische und gefühlsmäBige Verarbeitung von Informationen verstanden, d. h. als eine ganzheitliche, bewusste und unbewusste, intentionale und beiläufige, theoretische und praktische Verarbeitung von jener Art von Reizen, Eindrücken, Informationen, Begegnungen, Erlebnissen, Bedrohungen, Anforderungssituationen, symbolischen Präsentationen, virtuellen Umwelten etc., die 
aus der Umwelt auf den Menschen zukommen und von ihm wahrgenommen wer-

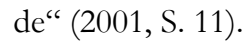

Es vollzieht sich eine Entgrenzung des Lernens in Richtung informeller Lernprozesse (vgl. Gruber 2007, S. 7).

Eine umfassende Definition der Konzepte des informellen Lernens und des Lernens in informellen Kontexten kann in diesem Beitrag nicht gegeben werden - zu vielfältig und widersprüchlich ist hier die Debatte ${ }^{1}$. Dennoch müssen zum besseren Verständnis einige Aspekte verdeutlicht werden.

Eine umfassende Definition des Begriffes informelles Lernen gestaltet sich deshalb so schwierig, da vielfach Definitionen Überschneidungen und Zusammenhänge mit anderen Lernformen, wie problemorientiertem, selbstgesteuerten, inzidentellem Lernen oder dem Lernen „en passant“ (vgl. Reischmann 1995) beinhalten. Eine der am weitesten verbreiteten Definitionen ist die Dreiteilung des Lernens, welche die Europäische Kommission vorschlägt: Sie unterteilt Lernprozesse in Bezug auf ihre institutionelle Verankerung in Formales Lernen, welches üblicherweise in „klassischen“ Bildungs- oder Ausbildungseinrichtungen zum Einsatz kommt und üblicherweise zertifizierbar ist, Nicht-formales Lernen, welches ebenso wie das Formale Lernen in Bezug auf Lernziele, -dauer und -mittel strukturiert ist, aber außerhalb dieser Einrichtungen stattfindet und gewöhnlich nicht zertifizierbar ist, und schließlich Informelles Lernen, welches weder in einer Bildungseinrichtung stattfindet noch (von außen) strukturiert ist oder zertifiziert wird (vgl. Dohmen 2001). Pietraß et al. zählen auch ,mediale Angebote, beispielsweise E-Learning-Programme - die einer didaktischen Vorstrukturierung unterliegen zu den informellen Lernmöglichkeiten, sofern sie nicht im Rahmen eines klar umrissenen (Weiter-)Bildungsangebots zum Einsatz kommen" (2005, S. 413). Die Autoren fassen zusammen, dass sich informelle Lerngelegenheiten gleichermaßen im Alltag oder im Beruf (bzw. der Schule) ergeben können und dass diese - obschon oftmals nicht eindeutig bestimmt werden kann, mit welchem Grad an Bewusstheit Wissen aufgebaut wurde - in konkreten Anwendungssituationen oftmals von großer Bedeutung sind (vgl. ebd.). Auch Overwien schlägt zur Präzisierung des Konzeptes informellen Lernens ein Hinzufügen der Kontexte vor. „Wenn informelles Lernen am Organisationsgrad des Lernens festgemacht wird, bleibt es immerhin nicht zwangsläufig eine Restkategorie, sondern wird als ein Feld deutlich, das durch verschiedene Lernorte mit unterschiedlichem Organisationsgrad des Lernens, gekennzeichnet ist" (Overwien 2001, S. 364).

\section{Qualitätsanforderungen für Lehr- Lern-Prozesse und Fazit}

Iske et al. erforschen am Kompetenzzentrum informelle Bildung (KIB) Qualitätsanforderungen von NutzerInnen an Online-Angebote. Sie schlagen zur Bestimmung dieser drei Faktoren vor: das Angebot selbst, die NutzerInnen und die Nutzungsgewohnheiten dieser. Ausgehend von diesem drei Bereichen identifizieren sie drei Ebenen der Passung, die für die Qualität eines Angebots ausschlaggebend sind: „Hierbei definiert

- die mediale Passung, ob die Formen des Online-Angebots,

- die inhaltliche Passung, ob Art und Thematisierung der Inhalte, und

- die interpersonale Passung, ob weitere Personen, die in das Angebot involviert

sind, den Vorstellungen, Interessen und praktisch realisierbaren Möglichkeiten unterschiedlicher NutzerInnen entsprechen" (Iske et al. 2007, S. 88). Die Passung eines Angebots auf eine/n spezifische/n NutzerIn und deren/dessen Gewohnheiten kann individuell als angenehm, gerade noch auszuhalten oder als Ausstiegsgrund empfunden werden.

Die Qualitätsanforderungen sind auch auf den Bildungsbereich zu übertragen. Die bisherigen Ausführungen haben gezeigt, dass es wenig sinnvoll ist E-Learning-Angebote (insbesondere im Internet) top-down einzuführen, ohne auf die individuellen Erfahrungen, Vorstellungen und Wünsche derjenigen zu achten, die anschließend damit lernen sollen. Es konnte auch deutlich gemacht werden, dass das Internet und in diesem befindliche E-Learning-Angebote, mehr als alle anderen Bildungsangebote, dazu in der Lage sind individuelle Lernumgebungen $\mathrm{zu}$ schaffen und auf persönliche Vorlieben oder Vorbehalte zu reagieren.

Aus diesem Grund wird dafür plädiert E-Learning-Angebote nicht mehr isoliert von aktuellen Forschungsergebnissen zu entwickeln, sondern im Gegenteil diese mit einzubeziehen. Weiterhin ist eine Chance in den Potenzialen informeller Lernprozesse zu sehen. So sollte hier ein verstärktes Augenmerk der Forschung liegen um am Beispiel der Prozesse, die den größten Teil menschlichen Lernens ausmachen (Faure 1973, S. 41, zit. n. Overwien 2001, S. 359) und im Internet ,ganz einfach“ und nebenbei ablaufen, selbst etwas zu lernen - nämlich wie Lernumgebungen optimaler Weise gestaltet sein sollten um Bildungsprozesse für alle Alters- und soziokulturellen Gruppen gleichermaßen interessant und brauchbar zu gestalten. 


\section{Anmerkung}

1 Für eine umfassende Beschäftigung mit der Debatte um diese Konzepte vgl. u.a. Dehnbostel (2010), Zürcher (2007), Overwien (2004, 2001), Dohmen (2001).

\section{Literatur}

Albrecht, R./ Frommann, U. / Phan Tan, T.T. (2005): Integrierte e-Learning-Services als Grundlage von qualifiziertem e-Learning an Hochschulen: Das Beispiel HannoverBraunschweig In: Zeitschrift für Hochschuldidaktik, No. 3, S. 64-79.

Barron, T. (2002): Trends in Corporate E-Learning. In: Rieckhof, H. C. \& Schüle, H.: E-Learning in der Praxis: Strategien, Konzepte, Fallstudien. Wiesbaden, S. 55-70.

Brinkmann, D. (2000): Moderne Lernformen und Lerntechniken in der Erwachsenenbildung: Formen Selbstgesteuerten Lernens. Bielefeld.

Close R. C., Humphreys R. \& Ruttenbur B. W. (2000): E-Learning and Knowledge Technology - Technology and the Internet are Changing the Way We Learn. Im Internet: http://www.masie.com/masie / researchreports/e-learn0.pdf (18.07.2011)

Dehnbostel, Peter; Seidel, Sabine; StammRiemer, Ida (2010): Einbeziehung von Ergebnissen informellen Lernens in den DQR eine Kurzexpertise. Helmut-SchmidtUniversität - Universität der Bundeswehr Hamburg, HIS Hochschul-InformationsSystem GmbH, Institut für Entwicklungsplanung und Strukturforschung GmbH. Bonn, Hannover. Online verfügbar unter http:// ankom.his.de/material/dokumente/ Expertise_Dehnbostel_StammRiemer_Seidel _2010.pdf? P H PSESS ID = a ed c 5 ef 6 abed63f301104cae794a50e6, zuletzt geprüft am 27.07.2011.

Dohmen, Günther (2001): Das informelle Lernen. Die internationale Erschließung einer bisher vernachlässigten Grundform menschlichen Lernens für das lebenslange Lernen aller. Herausgegeben von Bundesministerium für Bildung und Forschung (BMBF).

Gruber, E. (2007): Erwachsenenbildung und die Leitidee des lebenslangen Lernens. Magazin Erwachsenenbildung.at. Wien. Im Internet: http://erwachsenenbildung.at/magazin/? aid $=602 \& \mathrm{mid}=571$ [23.07.2011]

Hartmann, Maren/ Hepp, Andreas (Hrsg.) (2010): Die Mediatisierung der Alltagswelt. 1. Auflage. Wiesbaden: VS Verlag für Sozialwissenschaften (GWV).

Hasebrink, Uwe/ Domeyer, Hanna (2010): Zum Wandel von Informationsrepertoires in konvergierenden Medienumgebungen. In: Hartmann, Maren/ Hepp, Andreas (Hrsg.):
Die Mediatisierung der Alltagswelt. 1. Auflage. Wiesbaden: VS Verlag für Sozialwissenschaften (GWV), S. 49-64.

Hauske, S./ Bendel, O. (2007): Informelles E-Learning. Im Internet: http:// www.informelles-lernen.de/fileadmin/ dateien/Informelles_Lernen/Texte / Hauske_Bendel_2007.pdf (20.07.2011).

Iske, Stefan/ Klein, Alexandra/ Kutscher, Nadia/ Otto, Hans-Uwe (2007): Virtuelle Ungleichheit und informelle Bildung. Eine empirische Analyse der Internetnutzung Jugendlicher und ihre Bedeutung für Bildung und gesellschaftliche Teilhabe. In: Kutscher, Nadia/ Otto, Hans-Uwe/ Klein, Alexandra/ Iske, Stefan (Hrsg.): Grenzenlose Cyberwelt? Zum Verhältnis digitaler Ungleichheit und neuen Bildungszugängen für Jugendliche. 1. Auflage. Wiesbaden: VS Verlag für Sozialwissenschaften (GWV), S. 65-91.

Kerres, M./ Preussler, A./ Ojstersek N./ Stratmann, J. (2009): E-LearningUmgebungen in der Hochschule. In: Dittler, U. et al. (Hrsg.): E-Learning: Eine Zwischenbilanz. Kritischer Rückblick als Basis eines Aufbruchs. Münster, S. 101-116.

Kim, W./ Shih, T. K. (2003): Distance Education: The Status and Challenges. In: Journal of Object Technology, Volume 2, No. 6, S. 35-43.

Kubicek, H./ Breiter, A./ Fischer, A./ Wiedwald, C. (2004): Organisatorische Einbettung von E-Learning an deutschen Hochschulen. Institut für Informationsmanagement (ifib): Bremen.

Kutscher, Nadia/ Otto, Hans-Uwe/ Klein, Alexandra et al. (Hrsg.) (2007): Grenzenlose Cyberwelt? Zum Verhältnis digitaler Ungleichheit und neuen Bildungszugängen für Jugendliche. 1. Auflage. Wiesbaden: VS Verlag für Sozialwissenschaften (GWV).

Myrach, Th./ Montandon, C. (2008): Blended Learning. Kombinationen von Präsenzlehre und E-Learning. In: Thom, N./ Zaugg, R. J. (Hrsg.): Moderne Personalentwicklung. Mitarbeiterpotenziale erkennen, entwickeln und fördern. Wiesbaden, S. 191-206.

Otto, Hans-Uwe/ Coelen, Thomas (Hrsg.) (2004): Grundbegriffe der Ganztagsbildung. Beiträge zu einem neuen Bildungsverständnis in der Wissensgesellschaft. 1. Auflage. Wiesbaden: VS Verlag für Sozialwissenschaften.

Overwien, Bernd (2001): Debatten, Begriffsbestimmungen und Forschungsansätze zum informellen Lernen und zum Erfahrungslernen. In: Senatsverwaltung für Arbeit, Soziales und Frauen (Hrsg.): Tagungsband zum Kongreß „Der flexible Mensch“. Berlin: BBJVerlag, S. 359-376. 
Overwien, Bernd (2004): Internationale Sichtweisen auf „informelles Lernen“ am Übergang zum 21. Jahrhundert. In: Otto, Hans-Uwe/ Coelen, Thomas (Hrsg.): Grundbegriffe der Ganztagsbildung. Beiträge zu einem neuen Bildungsverständnis in der Wissensgesellschaft. 1. Auflage. Wiesbaden: VS Verlag für Sozialwissenschaften, S. 51-73.

Overwien, Bernd (2007): Informelles Lernen in der internationalen Diskussion. Online verfügbar unter http://www.informelleslernen.de/fileadmin/dateien/ Informelles_Lernen/Texte/ Overwien_2007.pdf, zuletzt geprüft am 27.07.2011.

Pietraß, Manuela/ Schmidt, Bernhard/ Tippelt, Rudolf (2005): Informelles Lernen und Medienbildung. Zur Bedeutung soziokultureller Voraussetzungen. In: Zeitschrift für Erziehungswissenschaft, Jg. 8, H. 3, S. 412-426.

Reischmann, Jost (1995): Die Kehrseite der Professionalisierung in der Erwachsenenbildung. Lernen „en passant“ - die vergessene Dimension. In: Grundlagen der Weiterbildung (GdWZ), H. 6, S. 200-204.

Rieckhof, H. C./ Schüle, H. (2002): Die Nutzung von E-Learning-Content in den Top-350 Unternehmen der deutschen Wirtschaft. In: Rieckhof, H. C./ Schüle, H. (Hrsg.): E-Learning in der Praxis: Strategien, Konzepte, Fallstudien. Wiesbaden, S. 133-162.

Senatsverwaltung für Arbeit, Soziales und Frauen (Hrsg.) (2001): Tagungsband zum Kongress „Der flexible Mensch“. Berlin: BBJVerlag.

Wache, Michael (2003): E-Learning - Bildung im digitalen Zeitalter. Herausgegeben von Bundeszentrale für politische Bildung. Online verfügbar unter http://www.bpb.de/files/ FWQFK9.pdf, zuletzt geprüft am 27.07.2011.

Zauchner, S./ Bauer, R./ Zobel, A./ Hupfer, M./ Herber, E./ Baumgartner, P. (2010): Technologien für lebenslanges Lernen - Wie eine Ära nach Learning-ManagementSystemen aussehen könnte. In: Tomaschek, N./ Gornik, E. (Hrsg.): The Lifelong Learning University - Perspektiven für die Universität der Zukunft, S. 61-70.

Zürcher, Reinhard (2007): Informelles Lernen und der Erwerb von Kompetenzen. Theoretische, didaktische und politische Aspekte. Herausgegeben von Kunst und Kultur (BMUKK) Bundesministerium für Unterricht.

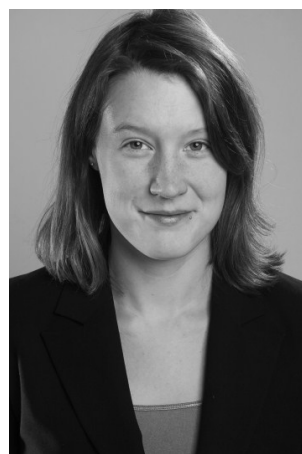

Jane Fleischer

Jg. 1985, Studium der Kommunikations- und Medienwissenschaft, Soziologie und Erziehungswissenschaft an der Universität Leipzig und der Universitá deglí studi di Palermo, seit 2010 wissenschaftliche Mitarbeiterin am Institut für Medien und Bildungstechnologie (imb) der Universität Augsburg, Professur für Digitale Medien.

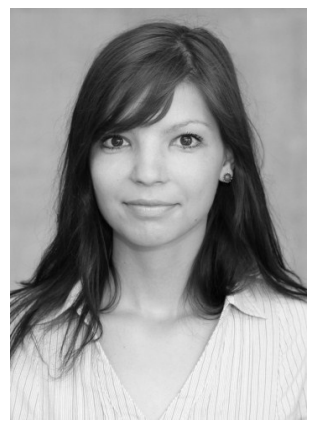

\section{Julia Hünniger}

Jg. 1984, Studium der Erziehungswissenschaft, Schwerpunkt Medienpädagogik und Interkulturelle Wirtschaftskommunikation an der Universität Jena und dem Dublin Institute of Technology, 2009-2010 Referentin für E-Learning an der Georg-Simon-Ohm-Hochschule für angewandte Wissenschaften Nürnberg, seit 2010 wissenschaftliche Mitarbeiterin am Institut für Medien und Bildungstechnologie (imb) der Universität Augsburg, Professur für Digitale Medien. 